\title{
Influence of nitric oxide-mediated vasodilation on the blood pressure measured with the tail-cuff method in the rat
}

\author{
Mariana Fritz ${ }^{1}$ \& Gustavo Rinaldi ${ }^{1,2, *}$ \\ ${ }^{1}$ Facultad de Ciencias Exactas, Departamento de Ciencias Biológicas, Universidad Nacional de La Plata, \\ La Plata, Argentina; ${ }^{2}$ Centro de Investigaciones Cardiovasculares, Consejo Nacional de Investigaciones \\ Cientificas y Técnicas (CONICET), 60 y 120 Piso 2, La Plata, 1900, Argentina
}

Received 28 December 2006; accepted 26 June 2007

(C) 2007 National Science Council, Taipei

Key words: L-NAME, systolic blood pressure, nitric oxide, tail-cuff method

\begin{abstract}
Systolic blood pressure (SBP) is frequently measured in rats by the tail cuff method, which usually comprises pulse/flow disappearance and reappearance during cuff inflation (Inf) and deflation (Def), separated by an interval between cycles (IBC). Although Def values are habitually used to estimate SBP, in 58 Wistar rats we found (Def-Inf) to be $-6 \pm 1 \mathrm{mmHg}$, indicating that Def $<$ Inf in most cases. When the IBC was lengthened to $2 \mathrm{~min}$, (Def-Inf) was increased to $-17 \pm 2 \mathrm{mmHg}$, indicating the probable accumulation of a vasodilating metabolite. This increase of (Def-Inf) was prevented by papaverine, indicating its relation to smooth muscle contractility. Adrenergic blockade did not prevent the increase of (Def-Inf), but pretreatment with L-NAME decreased it to $-5 \pm 2 \mathrm{mmHg}(p<0.05)$. Simultaneous measurement of SBP by tailcuff method and carotid cannulation revealed that the Inf value was the most accurate estimation of intravascular SBP. We conclude that: (1) the Inf value should be taken as representative of SBP, since depending on the duration of suprasystolic compression the Def value can underestimate it, and (2) nitric oxide accumulation due to flow deprivation was the main cause of SBP underestimation by Def values.
\end{abstract}

\section{Introduction}

Despite its shortcomings, the tail-cuff method for measuring systolic blood pressure (SBP) in rats is still very useful, especially when the screening of large populations of animals and/or the follow-up of SBP over long time periods are involved [1]. Several methods of flow/pulse detection have been described in addition to the original water plethysmograph [2] or modifications thereof [3-6]: mercury-in-rubber plethysmograph [7], Doppler effect [8-11], photoelectric [12-15], impedance [16], microphonic [17-19], and piezoelectric pulse detectors [20-23], this last being the most widely employed.

*To whom correspondence should be addressed. Fax: + 54-2214834833; E-mail: rinaldi@biol.unlp.edu.ar
With most of these methods it is possible to detect two events associated with the SBP: (1) disappearance of the pulse/flow signal during cuff inflation (Inf), and (2) its reappearance during deflation (Def).

However, in a revision of the bibliography specifically related to methodology of BP measurement, pulse/flow reappearance is the value overwhelmingly taken as representative of SBP [3, $7,12,24]$, even in papers which performed and reported both readings [25-28], but no reason was given for this election.

This would be a non-important matter if both values were equal, but in our laboratory's experience the readings obtained during the Def cycle are lower than the ones recorded during the Inf cycle. Careful inspection of the figures presented in some papers $[8,21,25,27]$ reveals the same fact. However, we found only two papers that specifically 
discussed differences between both measurements, one of them stating that the SBP values obtained during Inf and Def cycles were not statistically different [29]. In the other, however, the authors reported that the BP obtained during the Inf cycle is on the average $11 \mathrm{mmHg}$ higher that the one measured during Def, and the difference was statistically significant [5]. They also found that the values during the Inf cycle are in closer agreement with the intraarterial measurements than the Def ones. These data are coincident with our own results, but the authors did not provide any hypothesis and/or pursue further experiments to explain the difference between Inf and Def values [5].

Since the information on this subject seems to be scarce and contradictory, we undertook experiments destined to find out: (1) if the BP values obtained with the tail-cuff method are coincident during the Inf and Def cycles, and (2) In case of a lack of coincidence, which of the values better represents the intra-arterial readings, and the reason for the difference. Our hypothesis was that a vasodilator accumulated in the tail during the Inf-Def interval was responsible for the lower SBP values obtained during Def.

\section{Methods}

\section{Animals}

A total of 164 Wistar rats weighing 200-250 g were used in the course of this study. The animals were housed in cages with food and water adlibitum, complying with standard guidelines for care and use of laboratory animals.

\section{BP measurement}

For indirect SBP readings, the rats were placed in a chamber at $37^{\circ} \mathrm{C}$ for $10 \mathrm{~min}$, and then transferred to a standard setup with heating pad and acrylic restrainer, tail cuff and pulse sensor (Narco Biosystems, Houston, TX). The tail cuff was connected to a cylinder of compressed air through an arrangement of inlet and outlet valves that permitted Inf and Def of the cuff at a constant rate. The tail cuff pressure was continuously recorded with a solid state pressure sensor (Sensym, Honeywell Sensing \& Control, Inc.). The signals from the pulse and pressure sensors were conveniently amplified and then digitized with an analog-digital board (DT16EZ, Data Translation, Inc., Marlboro, MA) mounted in a desktop computer. On-line display for controlling the procedure, and files for later processing, were obtained with an appropriate software (Labtech Notebook Pro, Laboratory Technology Corp., Wilmington, MA). The indirect BP was always recorded during the Inf and Def cycles, and the interval of time between cycles (IBC) could be changed as desired. The indirect measurements were all performed by the same person, who was kept blind about the purpose of the study.

For direct BP measurement, the rats were anesthetized with sodium pentobarbital $(50 \mathrm{mg} /$ $\mathrm{kg}$ I.P.) and placed on a heating pad. The left carotid artery was exposed and cannulated with a short microbore teflon tubing $0.75 \mathrm{~mm}$ O.D. (Small Parts, Inc, Miami Lakes, FL) connected with a low-volume displacement pressure transducer (P23 Gb, Gould Inc., Cleveland, $\mathrm{OH}$ ). When the intraarterial tracing was stable, the tail cuff and pulse sensor were placed on the tail, and the indirect BP was measured at the same time. All the signals were sent to the same acquisition system (see above) so that both the indirect and direct BP tracings could be superimposed (see also Figure 1).

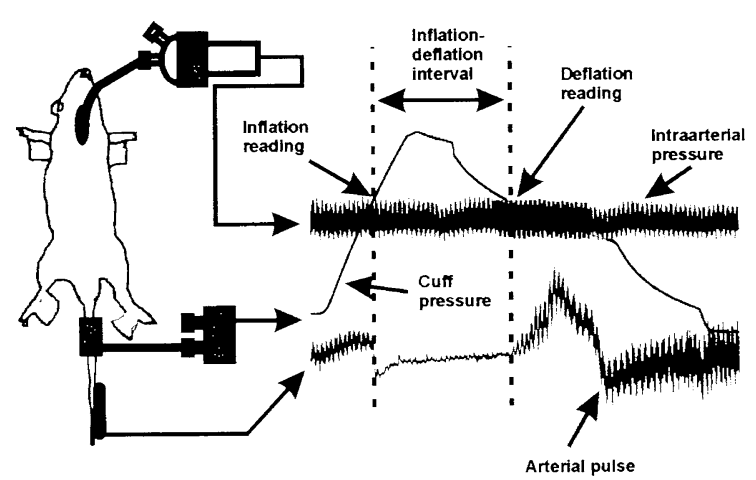

Figure 1. Left: schematic arrangement for direct and indirect measurement of BP. Right: actual recordings of simultaneous intraarterial and tail-cuff measurements, showing the variables referred to throughout the text. It should be noted that this setup was used only in 11 rats (see Figure 3); in the remaining experiments only the indirect SBP in conscious rats was used. 


\section{Histochemical NOS activity}

The NADPH-diaphorase assay was used to estimate total NOS activity in tail arteries removed after anesthesia with sodium pentobarbital $(50 \mathrm{mg} / \mathrm{kg}$ I.P.) under conditions that simulated as closely as possible the experimental situations compared. In the control experiments the tail was not compressed and the arteries were excised and dissected free of fat and connective tissue in a Petri dish filled with saline solution bubbled with a mixture of $5 \% \mathrm{CO}_{2}-95 \% \mathrm{O}_{2}$. In the "ischemic" experiments the tail was compressed with the cuff maintained at $180 \mathrm{mmHg}$ for $2 \mathrm{~min}$. The arteries were then rapidly excised and dissected free of fat and connective tissue in a Petri dish filled with saline solution bubbled with $\mathrm{N}_{2}$. All the vessels were fixed with $4 \%$ paraformaldehyde in $0.1 \mathrm{M}$ phosphate buffer ( $\mathrm{pH} 7.4$ ); cryoprotected in $15 \%$ sucrose and frozen. Sections of $15 \mu \mathrm{m}$ were cut on a cryostat, mounted on gelatin-coated glass slides, and incubated with a reaction mixture containing $1.2 \mathrm{mM}$ NADPH and $0.24 \mathrm{mM}$ nitro blue tetrazolium in phosphate buffer added with $0.3 \%$ Triton X-100 for $60 \mathrm{~min}$ at $37^{\circ} \mathrm{C}$. The NOS activities assayed by this technique are inhibited by preincubation with diphenyleneiodonium and other NOS inhibitors. A Zeiss Axiophot microscope was used for observation, absorption determination, and photography. Computerized image analysis was performed using an analyzer (Kontron Zeiss), and mean absorption values were calculated from five areas of each section and from 10 different sections. The determinations were performed under similar conditions of light, gain, offset, and magnification. The technician was kept blind about the purpose of the measurements. No reaction product was found when NADPH was omitted. In control experiments, $5 \mathrm{mM}$ L-NAME was added to consider the specific NADPHdiaphorase staining due to NOS activity.

\section{Estimation of vascular superoxide anion $\left(\cdot \mathrm{O}^{2-}\right)$ production}

$\cdot \mathrm{O}^{2-}$ production was measured using lucigenin chemiluminescence. Briefly, after preparation in the conditions described for histochemical NOS activity, the vessels were placed in a modified Krebs/Hepes buffer and allowed to equilibrate for $30 \mathrm{~min}$ at $37^{\circ} \mathrm{C}$. Scintillation vials containing $2 \mathrm{ml}$
Krebs/Hepes buffer with $5 \mu \mathrm{M}$ lucigenin were placed into a scintillation counter (Chameleon, Hidex Co., Finland) switched to the out-of-coincidence mode. After dark adaptation, background counts were recorded and a vascular segment was added to the vial. Scintillation counts were then recorded every $30 \mathrm{~s}$ for $15 \mathrm{~min}$, and the respective background counts were substracted. The vessels were then dried by placing them in a $90{ }^{\circ} \mathrm{C}$ oven for $2 \mathrm{~h}$, for determination of dry weight. Lucigen counts were calculated as counts $\cdot 10^{3}$ per min per mg dry weight.

Drugs: papaverine, propranolol, phenoxybenzamine, L-NAME and other analytical chemicals were purchased from SIGMA (St. Louis, MO).

The results were expressed as mean \pm 1 S.E.M., and the differences between means were evaluated using a statistical package (Sigmastat 2.0, Jandel Scientific Software, San Rafael, CA). When only two groups were involved the Student's $t$ test (paired or unpaired) was used, while for multiple groups comparisons the one-way ANOVA was employed. The particular modifications of these tests as dictated by the circumstances are indicated in Methods for each specific protocol. The correlation between variables was studied with linear regression.
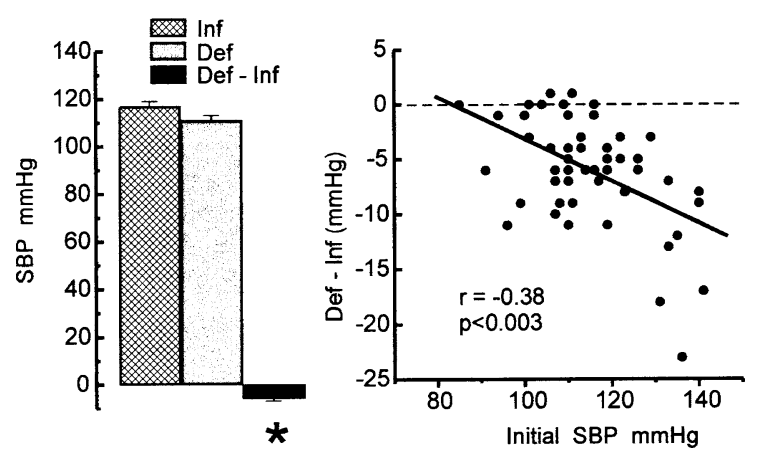

Figure 2. Left panel: SBP measured by the tail-cuff method in 58 Wistar rats, showing the Inf and Def readings and the difference between them (Def-Inf), which is significant. The InfDef interval was $\cong 30 \mathrm{~s}$. Right panel: scatter graph plotting (Def-Inf) as a function of the initial SBP (i.e. the Inf reading) for each animal, showing a significant correlation between both variables. ${ }^{*}=p<0.05$. 


\section{Results}

Figure 2 depicts on the left panel the SBP measured during the Inf and Def cycles in 58 Wistar rats, showing that the Def readings were lower than their Inf counterparts. The average difference between Def and Inf values (Def-Inf) was slight $(-6 \pm 1 \mathrm{mmHg})$, although significant $(p<0.001$, Wilcoxon Signed Rank Test). The right panel shows the dependence of the (Def-Inf) on the prevailing SBP, and it can be seen that in only seven rats the Def reading was equal to, or greater than, the Inf counterpart $(r=-0.38, p<0.003$, Pearson product moment correlation). These were routine measurements of $\mathrm{BP}$ that had been performed recently in our laboratory, in which no particular attention was paid to the duration of the IBC. When the records were revised afterwards, the IBC had been around $30 \mathrm{~s}$.

In 11 additional experiments in Wistar rats (Figure 3) we performed simultaneous measurement of BP by the tail-cuff method and by direct carotid cannulation, and we obtained similar values for the direct SBP and the Inf readings (NS, Wilcoxon Signed Rank Test). However, the Def reading was slightly but significantly lower than the directly measured SBP $(p<0.006$, paired $t$ test). Since these were the only experiments performed

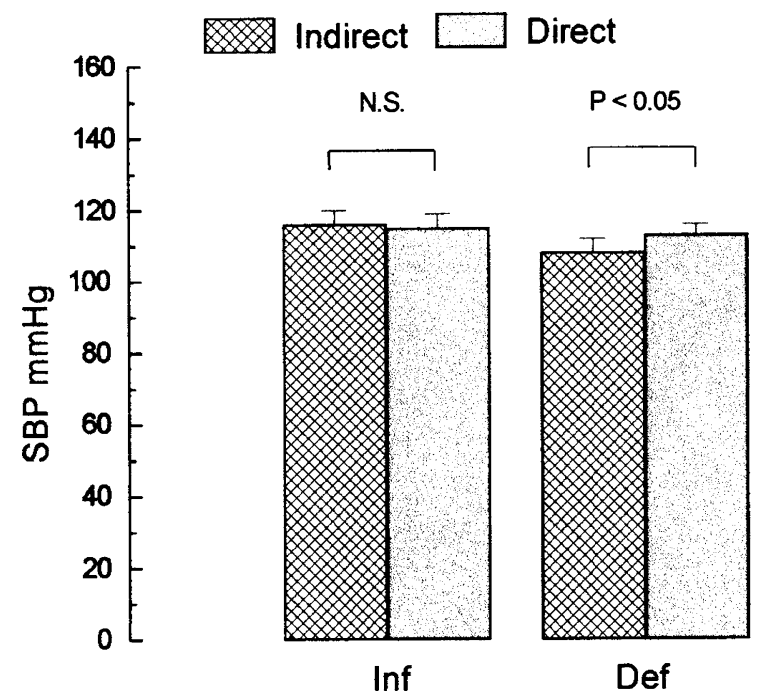

Figure 3. SBP measured simultaneously by the tail-cuff method (Inf and Def readings) and by carotid cannulation in 11 Wistar rats. The Def readings were slightly, although significantly, lower than the direct measurement; hence the Inf reading estimated the intraarterial SBP more accurately. with anesthesia, we compared the indirect $\mathrm{BP}$ values with the ones obtained in the same rats before surgery, and we found no significant differences between them (conscious rats: $115 \pm 2 \mathrm{mmHg}$, anesthetized rats: $116 \pm 4 \mathrm{mmHg}, n=11$, NS with the paired $t$ test) (data not illustrated).

To evaluate the relationship between (Def-Inf) and the duration of the IBC we measured the SBP in 12 Wistar rats by the tail-cuff method in two consecutive runs (Figure 4): in the first one the IBC was less than $15 \mathrm{~s}$ (left bars), while in the second the IBC was increased to 2 min (middle bars). As can be seen, the lengthening of the IBC produced a significant increase of (Def-Inf) to $-15 \pm 2 \mathrm{mmHg}(p<0.001$, paired $t$ test $)$.

The influence of a nonspecific smooth muscle dilator, papaverine, was evaluated in seven of the previous Wistar rats in which a third SBP measurement was performed $20 \mathrm{~min}$ after the administration of $75 \mathrm{mg} / \mathrm{kg}$ I.P. of papaverine and also keeping the IBC in $2 \mathrm{~min}$. As can be seen in the right bars on Figure 4 the (Def-Inf) was significantly reduced by papaverine and returned to values not different from zero $(p=0.69$, paired $t$ test).

In another set of experiments (Figure 5) we evaluated the participation of two important routes of vascular smooth muscle control, i.e. the sympathetic innervation and the NO pathway, on the

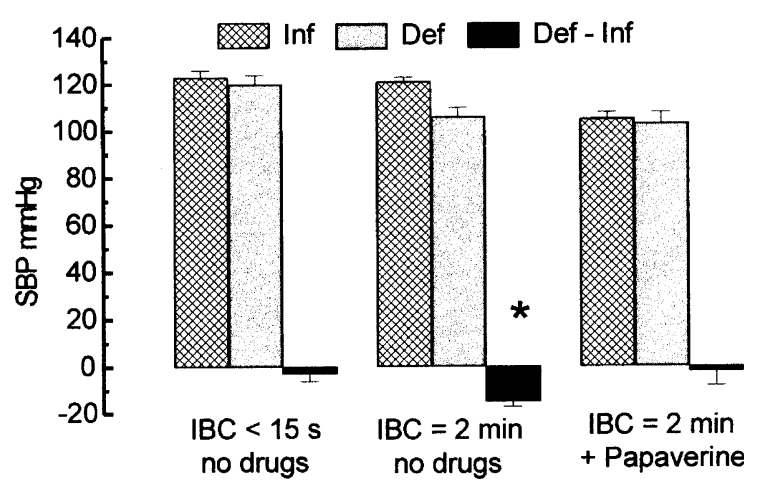

Figure 4. Effect of the length of the interval between the Inf and Def cycles (IBC) on the (Def-Inf). The bars on the left represent 12 measurements in which the IBC was less than $15 \mathrm{~s}$, and the (Def-Inf) was nearly zero. The bars on the middle represent 12 measurements in which the IBC was increased to $2 \mathrm{~min}$, and in this case the (Def-Inf) augmented significantly. The bars on the right represent seven measurements in which the IBC was also increased to $2 \mathrm{~min}$, but with the previous administration of $75 \mathrm{mg} / \mathrm{kg}$ of papaverine i.p. $(n=7)$, and in this case the increment of the (Def-Inf) was prevented. $*=p<0.05$ with respect to zero. 


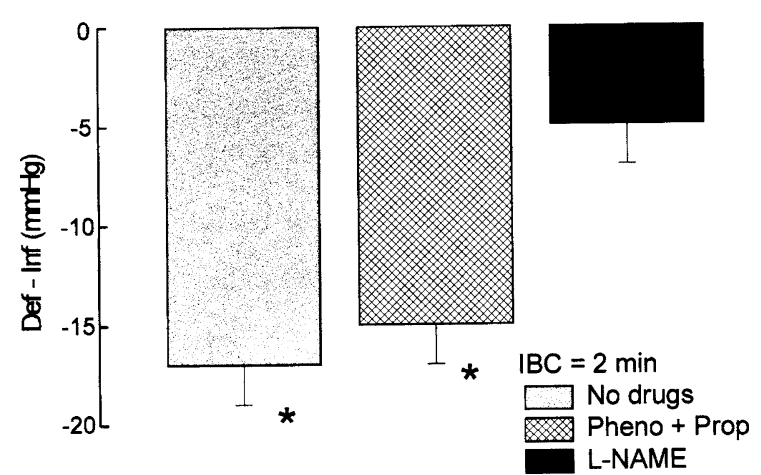

Figure 5. Experiments in which the IBC was kept deliberately at $2 \mathrm{~min}$. In absence of drugs (left bar, $n=21$ ) the (Def-Inf) was significant $(-17 \pm 2 \mathrm{mmHg}, p<0.05)$. Administration of phenoxybenzamine and propranolol (middle bar, $n=8$ ) failed to reduce the (Def-Inf), which remained at $-15 \pm 2 \mathrm{mmHg}$. The right bar represents the same measurement in rats pretreated with L-NAME in the drinking water for 3 weeks $(n=10)$, and in this case the (Def-Inf) was significantly reduced to $-5 \pm 2 \mathrm{mmHg}$. $*=p<0.05$ with respect to zero.

increase of (Def-Inf). In 17 rats with no drugs the IBC was fixed in $2 \mathrm{~min}$, obtaining a significant (Def-Inf) (left bar, $p<0.001$, paired $t$ test). Administration of phenoxybenzamine $(4 \mathrm{mg} / \mathrm{kg}$ i.p.) and propranolol $(10 \mathrm{mg} / \mathrm{kg}$ i.p.) to eight of these rats produced an effective adrenergic blockade as could be ascertained by the fall in blood pressure from $103 \pm 2 \mathrm{mmHg}$ to $75 \pm 2 \mathrm{mmHg}$ (Inf readings); however, the Def cycle under adrenergic blockade produced still lower readings of $62 \pm 3 \mathrm{mmHg}$. This Def-Inf difference $(14 \pm 2 \mathrm{mmHg})$ was not significantly different from the one obtained in absence of adrenergic blockade $(-17 \pm 2 \mathrm{mmHg})$. The right bar represents the same measurement in 10 rats pretreated with L-NAME $70 \mathrm{mg} / \mathrm{kg}$ per day in the drinking water for three weeks. The blockade of NO synthesis was effective as could be ascertained by an increase in BP from $111 \pm 3 \mathrm{mmHg}$ to $123 \pm 3 \mathrm{mmHg}$ at the end of the treatment (Inf readings). However, the (Def-Inf) in presence of L-NAME was decreased to $-5 \pm 2 \mathrm{mmHg}$, a significant reduction with respect to the control group ( $p<0.05$, one-way ANOVA).

Additional experiments were designed to compare the effect of a continued compression of the tail versus fast, repeated Infs and Defs during the same time period, and to study if there was a correlation between both types of measurement and the magnitude of the (Def-Inf). Figure 6 shows in open circles the measurements with IBC

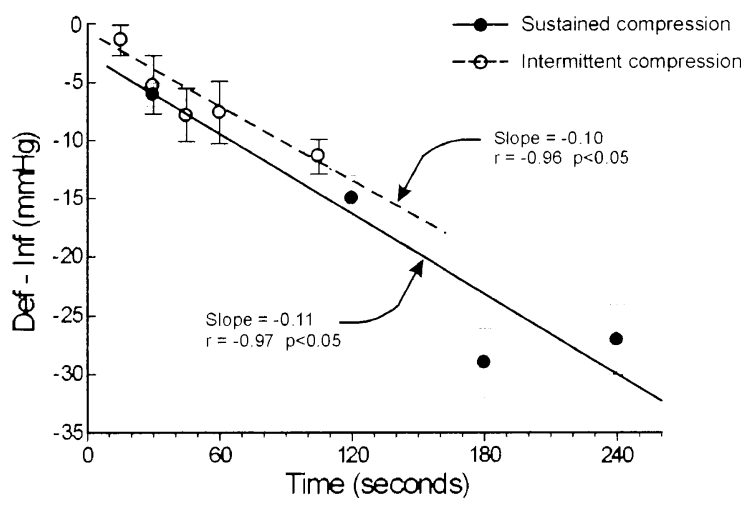

Figure 6. Experiments showing that both a continued tail compression (closed circles) and repeated short cycles of compression/decompression (open circles) can increase the (DefInf). The slopes of both regression lines are nearly identical. The number of experiments represented by each symbol are detailed in Methods. With the exception of the intermittent measurement at $15 \mathrm{~s}$, the rest of the (Def-Inf) values were significant $(p<0.05)$, although the asterisks were omitted next to each symbol for the sake of clarity.

$<15$ s repeated during $15,30,45,60$ and $105 \mathrm{~s}$ $(n=14$ in each time period), and it can be seen that a significant correlation exists between the duration of the repeated measurements and the magnitude of the (Def-Inf) $(r=-0.96, p<0.05$, Pearson product moment correlation). For comparison, in closer circles are shown measurements in which the tail compression was sustained during $30 \mathrm{~s} \quad(n=58), 120 \mathrm{~s} \quad(n=12), 180 \mathrm{~s}$ $(n=15)$ and $240 \mathrm{~s}(n=11)$, and the correlation between IBC and (Def-Inf) is also significant $(r=-0.97, p<0.05$, Pearson product moment correlation), although it should be noted that continued compression for longer than $180 \mathrm{~s}$ does not produce a greater (Def-Inf). With the exception of the intermittent measurement at $15 \mathrm{~s}$, the rest of the (Def-Inf) values shown in Figure 6 were significant (Kruskal-Wallis One Way Analysis of Variance on Ranks with Dunnett's Method). It is also worth mentioning that intermittent compression and decompression could not be extended for more than $120 \mathrm{~s}$ due to animal discomfort and unrest; while continued compression could be extended for up to $240 \mathrm{~s}$ without signs of animal discomfort.

In 12 additional rats we used the NADPHdiaphorase assay to estimate total NOS activity in tail arteries excised in control conditions and after $2 \mathrm{~min}$. of ischemic compression. The histochemical analysis showed a significant increase in the 
absorption values from $0.1210 \pm 0.0042$ (control experiments) to $0.1539 \pm 0.0081$ (ischemic arteries) ( $p<0.05$, unpaired $t$ test).

In four additional experiments in which we measured $\cdot \mathrm{O}^{2-}$ production using lucigenin chemiluminescence we could not detect consistent differences between control and ischemic vessels (data not shown).

\section{Discussion}

Byrom and Wilson in 1938 [7] described an apparatus consisting of a manually-inflated cuff located at the base of the tail and a distal water plethysmograph to detect the reappearance of flow during Def. Although the procedure seemed to be cumbersome and required that the animals be anesthetized, Okamoto and Aoki still used this method 25 years later in their pioneer work on the development of a strain of spontaneously hypertensive rats [25]. With this method it was inevitable to estimate $\mathrm{BP}$ during the Def cycle at the point where the cuff pressure was just below the systolic BP but still above the venous pressure, thus allowing the plethysmograph to detect the subsequent increase in tail volume.

In contrast, all the methods of pulse detection described thereafter, i.e. mercury-in-rubber plethysmograph [7], Doppler effect [8-11], photoelectric [12-15], impedance [16], microphonic [17-19], and piezoelectric pulse detectors [20-23], permitted to take Inf and Def readings, but the Def values were overwhelmingly used to estimate SBP. Usually no reasons were given for this election, although for example Buñag [29] indicated his preference for the Def cycle because the animal was more mobile during the Inf cycle and the possibility of an erroneous reading due to noisy signals was increased.

Taking advantage of the incorporation of digitizing techniques to our BP measuring setup we could easily retrieve several routine BP measurements made in the past and found that the Def cycle effectively yielded lower values than the Inf cycle: the (Def-Inf) was $-6 \pm 1 \mathrm{mmHg}$ in 58 Wistar rats. Even with repeated Inf-Def cycles the Def value was decreased, leading to an erroneous estimation of SBP. This finding is in disagreement with the report of Buñag [29] who measured both cycles and found them to be not significantly different. However, in this paper as well as in others $[8,21,25,27]$ close inspection of some representative tracings does show a lower BP value during Def. On the contrary, our measurements coincide with the paper of Martinelli et al. [5] who reported a significant decrease of $11 \mathrm{mmHg}$ for the Def cycle, nearly double than the one we found in normotensive rats. This difference can probably be accounted for by their method of pulse detection, a water plethysmograph, which probably implied a longer Inf-Def interval than in our experiments. When we subsequently performed simultaneous measurements of the BP by the tail-cuff method and by direct carotid cannulation we found that the Inf cycle matched the intravascular measurement; and this result is also in agreement with the findings of Martinelli et al. [5].

In analyzing the causes for this phenomenon, the simplest explanation could be that in the Inf cycle the cuff pressure has to be higher than the intravascular BP for the pulse to disappear, and the contrary holds true for the Def cycle. This in itself implies a lower estimation during Def if the intravascular BP is stable. However, even in this case the difference is likely to be too small to be detected with the current methods, and more so if the inevitable oscillations in $\mathrm{BP}$ over time are taken into account.

A purely physical explanation could be based on the different velocities of blood flow during the Inf and Def cycles. When the cuff is constricted, flow velocity progressively decreases to zero; but when the cuff deflates the arterial lumen is initially narrower than normal, and flow velocity is high. According to Bernoulli's theorem, if the fluid flows horizontally so that no change in gravitational potential energy occurs, then an increase in fluid velocity is associated with a decrease in fluid pressure and vice versa. The decrease of pressure against the vessel wall (i.e. the Venturi effect) could be reflected in a decrease of the cuff pressure necessary to counteract it, contributing to a difference between Def and Inf readings. This explanation would be compatible with our finding that the (Def-Inf) was significantly correlated with the prevailing BP: the greater the BP, the greater the velocity of blood flow upon reopening of the arterial lumen and the greater the (Def-Inf).

However, the foregoing explanation considers the tail artery as an entirely passive conduit, which 
is not the case. When during the Def cycle the cuff pressure ceases to counteract the intravascular BP, the state of constriction of vascular smooth muscle constitutes the principal remaining resistance to flow, and it must be overcome for the arterial pulse to reappear. By decreasing the smooth muscle tone during Def, the readings obtained could appear lower than the ones obtained during Inf. This lowered smooth muscle tone could be provoked by a vasodilator accumulated during the Inf-Def interval, when the tail is rendered ischemic.

Two pieces of experimental evidence support this possibility. Firstly, the experiments in which we extended the Inf-Def interval (and thus the ischemic period) to $2 \mathrm{~min}$ and obtained a corresponding increase in the (Def-Inf), which went up to $-17 \pm 2 \mathrm{mmHg}$. Secondly, the experiments in which we employed the nonspecific smooth muscle relaxant papaverine [30] to suppress smooth muscle tone and, contrariwise, obtained a decrease of the (Def-Inf). By placing the smooth muscle in a state of relaxation beforehand, we could prevent the action of the hypothesized vasodilator.

We then set out to investigate which vasodilator could participate in this phenomenon, focusing our attention on sympathetic innervation and on the nitric oxide (NO) system. For that purpose we used BP readings with a 2 min interval between Inf and Def cycles in order to turn the (Def-Inf) well evident; the first measurement was performed in control conditions (no blockers) and the second after the specific blocker had been administered. When the sympathetic system was explored, alphaand beta-blockade were induced simultaneously to make sure that the tail artery, usually richly innervated, did not receive any autonomic influence. Although the decrease in SBP indicated that the adrenergic blockade was successful, a significant (Def-Inf) still persisted, indicating that the sympathetic system was not relevant for the production of this phenomenon. It must be pointed out that the sympathetic blockade was probably more effective than could appear from the SBP values because the prevailing SBP had been decreased and this by itself lowers the (DefInf) as discussed before. Had the SBP remained stable, the (Def-Inf) would have been even greater.

Similar experiments were carried out in rats that had received L-NAME in the drinking water for the previous three weeks. In this case, the (DefInf) was decreased to non-significant values indi- cating that NO accumulation during the occlusion period was relevant in its production. It has to be noted that the blockade of NO synthesis was even more effective than could appear from the crude numbers because the prevailing SBP increased with the administration of L-NAME, and this by itself augments the (Def-Inf) as discussed before. Had the SBP remained stable, the decrease of (Def-Inf) would have been even greater.

Since these experiments provided only indirect evidence of the NO involvement in the phenomenon studied, we attempted to estimate histochemical NOS activity in the tail artery by the NADPHdiaphorase assay. These studies showed an increase of $28.5 \pm 9.9 \%$ of NOS activity in the samples obtained from vessels obtained after cuff compression and preserved under hypoxic conditions. Although the histochemical measurement does not differentiate between the three NOS isoforms, it is plausible that the increased activity corresponds to eNOS, the predominant isoform in normal vascular endothelium. The inducible form (iNOS) is unlikely to be stimulated in our experimental conditions (i.e. non-atherosclerotic vessels without macrophage proliferation).

Further mechanisms of NO accumulation during the ischemic interval could be the following: (1) since there is a tonic production and release of $\mathrm{NO}$ by the endothelium [31], and the cuff compression precludes either the arterial inflow and the venous outflow, NO is likely to accumulate and be responsible for the vasodilation during the Def cycle; and (2) endothelial cell deformation is another cause of NO production [31], but in this case it can probably be ruled out because during the ischemic interval there is no flow and consequently no shear stress. Upon restoration of flow there will be shear stress and NO release, but its effect would be evident later on, and not when the pulse reappears.

It is well known that the ischemia produces reactive oxygen species, among which the superoxide anion $\left(\cdot \mathrm{O}^{2-}\right)$ is of critical importance $[32,33]$. Many others, including $\mathrm{H}_{2} \mathrm{O}_{2}$, peroxynitrite, hypochlorous acid, the hydroxyl radical, and lipid radicals, are derived from $\mathrm{O}^{2-}$. Both superoxide and NO are highly reactive and unstable radicals, and react very rapidly to form the major product peroxynitrite. Although peroxynitrite can produce vasodilation, this effect occurs at concentrations far in excess of the effective vasorelaxant concen- 
trations of NO [34, 35] and probably was not important in our experiments. On the other hand, this superoxide/peroxynitrite production can reduce the bioavailability of NO because of oxidative inactivation [33], but this effect could not have explained the lengthening of the (Def-Inf), since it would be vasoconstricting and not vasodilating. At any rate, the lucigenin experiments failed to demonstrate a consistent difference between hypoxic and control arteries after 2 min of compression, which was probably too short a period to elicit an important $\cdot \mathrm{O}^{2-}$ production.

To our knowledge this is the first study looking for an explanation of the difference between Inf and Def values obtained during tail cuff measurement of SBP in rats, and establishing nitric oxide accumulation during the period of flow deprivation as the main cause of this difference.

The consequences of our data for the laboratory practice are evident: the Inf value should be taken as representative of SBP, and suprasystolic compression by the tail cuff should be kept as short as possible, since the error of SBP estimation can be quite important. Surely enough, experienced operators will tend to proceed in this way and then the error inherent to the Def reading would be small or even non-existent. But if lessexperienced persons and/or non-trained animals are involved, repeated attempts to measure $\mathrm{BP}$ will lead to prolonged tail compression and ischemia, as proved in this study, and would render Def readings that do not estimate intravascular SBP accurately.

\section{References}

1. Van Vliet V.N., Chafe L.L., Antic V., Schnyder-Candrian S. and Montani J.-P., Direct and indirect methods used to study arterial blood pressure. J. Pharmacol. Toxicol. Methods 44: 361-373, 2000.

2. Byrom F.B. and Wilson C., A plethysmographic method for measuring systolic blood pressure in the intact rat. J. Physiol. 93: 301-304, 1938.

3. Dodson L.F. and Mackaness G.B., The estimation of basal blood pressure in rat by tail oscilloscope. Br. J. Exp. Pathol. 38(6) 618-627, 1957.

4. Lucas J., A modified indirect method of blood pressure measurement in the conscious and anaesthetized rat. $\mathrm{J}$. Physiol. 218(1) 1P-3P, 1971.

5. Martinelli J.G., Beraldo P.S., Campos G.P. and da Costa e Silva A., A new plethysmograph for detecting arterial pulse waves in rat tail. Nephron 39(1) 61-63, 1985.
6. Sobin S.S., Accuracy of indirect determinations of blood pressure in the rat; relation to temperature of plethysmograph and width of cuff. Am. J. Physiol. 146: 179-186, 1946.

7. Maistrello I. and Matscher R., Measurement of systolic blood pressure of rats comparison of intraarterial and cuff values. J. Appl. Physiol. 26: 188-193, 1969.

8. Buñag R.D. and Riley E., Simultaneous measurements in awake rats of drug-induced changes in carotid and tail-cuff systolic pressures. J. Appl. Physiol. 36(5) 621-624, 1974.

9. Newman D.L. and Looker T., Simultaneous measurement of the systolic blood pressure and heart rate in the rat by a transcutaneous method. Lab. Anim. 6(2) 207-211, 1972.

10. Reichle F.A., Noninvasive blood pressure measurement in the rat by ultrasonic auscultation. J. Surg. Res. 11: 539544, 1971.

11. Rowberg A., Franklin D. and Van Citters R.L., Nontraumatic method for measurement of blood pressure in animals with tails. J. Appl. Physiol. 27: 301-302, 1969.

12. Hermansen K., A new method for determination of the systolic blood pressure in conscious rats. Life Sci. 9: 12331237, 1970.

13. Ikeda K., Nara Y. and Yamori Y., Systolic and mean blood pressure determination by a new tail cuff method in spontaneously hypertensive rats. Lab. Anim. 25(1) 26-29, 1991.

14. Lee R.P., Wang D., Lin N.T., Chou Y.W. and Chen H.I., Modified technique for tail cuff pressure measurement in unrestrained conscious rats. J. Biomed. Sci. 9(5) 424-427, 2002.

15. Palbøl J. and Henningsen J., Blood pressure measurements in the conscious rat. An improved photoelectric, sphygmomanometric device. Scand. J. Urol. Nephrol. 13(3) 319321, 1979.

16. Wen S.F., Tremblay J.M., Qu M.H. and Webster J.G., Impedance method for blood pressure measurement in awake rats without preheating. Hypertension 11(4) 371375, 1988.

17. Buñag R.D., Pressor effects of the tail-cuff method in awake normotensive and hypertensive rats. J. Lab. Clin. Med. 78(4) 675-682, 1971.

18. Fregly M.J., Factors affecting indirect determination of systolic blood pressure of rats. J. Lab. Clin. Med. 62: 223230, 1963.

19. Friedman M. and Freed S.C., Microphonic manometer for indirect determination of systolic blood pressure in the rat. Proc. Soc. Exp. Biol. Med. 70: 670-672, 1949.

20. Resurreccion A.V. and Caster W.O., Problems in the interpretation of blood pressure changes seen in rats fed a breakfast cereal. Life Sci. 22: 1821-1826, 1978.

21. Pfeffer J.M., Pfeffer M.A. and Frohlich E.D., Validity of an indirect tail-cuff method for determining systolic arterial pressure in unanesthetized normotensive and spontaneously hypertensive rats. J. Lab. Clin. Med. 78(6) 957-962, 1971.

22. Irvine R.J., White J. and Chan R., The influence of restraint on blood pressure in the rat. J. Pharmacol. Toxicol. Methods 38(3) 157-162, 1997.

23. Kusaka M., Kishi K. and Sokabe H., Does so-called streptozocin hypertension exist in rats? Hypertension 10(5) 517-521, 1987.

24. Bazil M.K., Krulan C. and Webb R.L., Monitoring of cardiovascular parameters in conscious spontaneously 
hypertensive rats. J. Cardiovasc. Pharmacol. 22(6) 897-905, 1993.

25. Buñag R.D., Mueting N. and Riley E., Regional vascular influences on tail-cuff measurements of drug-induced changes in systolic pressure. J. Appl. Physiol. 39: 724 727, 1975.

26. Yamakoshi K.I., Shimazu H. and Togawa T., Indirect measurement of instantaneous arterial blood pressure in the rat. Am. J. Physiol. 237(5) H632-H637, 1979.

27. Swales J.D. and Tange J.D., A photoelectric method of blood pressure measurement in the rat. J. Lab. Clin. Med. 75: 879-885, 1970.

28. Dowd D.A. and Jones D.R., A method for recording baby rat systolic blood pressures. J. Appl. Physiol. 25: 772-774, 1968.

29. Buñag R.D., Validation in awake rats of a tail-cuff method for measuring systolic pressure. J. Appl. Physiol. 34: 279282, 1973.

30. Needleman P. and Johnson M. Jr., Vasodilators and the treatment of angina, In: Goodman Gilman A., Goodmsn L.S. and Gilman A. (Eds.), Goodman and Gilmańs The Pharmacological Basis of Therapeutics, 6thth ed.. Macmillan Publishing Inc., Co, New York, 1980, pp. 830.
31. Moncada S. and Higgs E.A., The discovery of nitric oxide and its role in vascular biology. Br. J. Pharmacol. 147: S193-S201, 2006.

32. Münzel T., Afanas'ev I.B., Kleschyov A.L. and Harrison D.G., Detection of Superoxide in Vascular Tissue. tissues. Arterioscler. Thromb. Vasc. Biol. 22: 1761-1768, 2002.

33. Kojda G. and Harrison D., Interactions between NO and reactive oxygen species: patophysiological importance in atherosclerosis, hypertension, diabetes and heart failure. Cardiovasc. Res. 43: 562-571, 1999.

34. Villa L.M., Salas E., Darley-Usmar V.M., Radomski M.W. and Moncada S., Peroxynitrite induces both vasodilation and impaired vascular relaxation in the isolated perfused rat heart. Proc. Natl. Acad. Sci. USA 91: 12383-12387, 1994.

35. Liu S., Beckman J.S. and Ku D.D., Peroxynitrite, a product of superoxide and nitric oxide, produces coronary vasorelaxation in dogs. J. Pharmacol. Exp. Ther. 268: 1114-1121, 1994. 